\title{
Comment on Propagation and Dissipation of Alfvén Waves in Coronal Holes
}

\author{
Suresh Chandra*
}

School of Physical Sciences, S.R.T.M. University, Nanded 431 606, India

\begin{abstract}
Dwivedi and Srivastava [1] (DS) investigated the propagation and dissipation of Alfvén waves in coronal holes after accounting for the viscosity and magnetic diffusivity. After solving a set of equations with the help of computer results are reported by them. We find that the same set of equations can be solved even analytically. Since DS have not reported any values of physical parameters used by them except their expressions, we could not trace out the source of error. One reason for the difference in our results and those of DS can be assigned to some mistakes in their computer program or to the values of parameters used.
\end{abstract}

Keywords: MHD-waves-solar wind-sun, magnetic field.

\section{INTRODUCTION}

Since the pioneering work of Parker [2], a large amount of work has been done on the potential role of magnetohydrodynamics (MHD) waves for understanding the problems of coronal heating and the solar wind acceleration mechanisms. Measurement of increasing line width with altitude above the limb by Hassler [3] has been interpreted as the signature of outward propagating undamped Alfvén waves. On the other side, the narrowing of the line profile at higher altitude could serve as signature of Alfvén waves dissipation [4, 5]. For the study of the propagation and dissipation of Alfvén waves in coronal holes, DS considered the following MHD equations:

$\rho \frac{\partial \vec{v}}{\partial t}+\rho(\vec{v} \cdot \nabla) \vec{v}=\frac{1}{\mu}(\nabla \times \vec{B}) \times \vec{B}+\rho v \nabla \vec{v}$

$\frac{\partial \vec{B}}{\partial t}=\nabla \times(\vec{v} \times \vec{B})+\eta \nabla^{2} \vec{B}$

$\nabla \cdot \vec{B}=0$

where $\vec{v}$ is the velocity, $\vec{B}$ the magnetic field and $\rho, \mu, \eta, v$ are respectively the mass density, magnetic permeability, magnetic diffusivity and coefficient of viscosity. For these equations (1) - (3), dispersion relation is derived by Pekünlü et al. [5]:

$\omega^{2}=k^{2}\left[v_{A}^{2}-i \omega(v+\eta)\right]+v \eta k^{4}$

where $v_{\mathrm{A}}\left[=\mathrm{B}_{0} / \sqrt{\mu \rho 0}\right]$ is the Alfvén velocity. The wave number $k$ may be complex quantity and can be expressed as $k=k_{r}+i k_{i}$. DS considered two cases:

(i) There is the viscosity only. That is, there is no magnetic diffusivity $(\eta=0)$. For this case, equation (4) gives

*Address correspondence to this author at the School of Physical Sciences, S.R.T.M. University, Nanded 431 606, India; E-mail: suresh492000@yahoo.co.in
$k_{r}^{4}-P k_{r}^{2}-Q^{2}=0 \quad$ and $\quad k_{r} k_{i}=Q$

where

$$
P=\frac{\omega^{2} v_{A}^{2}}{v_{A}^{4}+\omega^{2} v^{2}} \quad \text { and } \quad Q=\frac{\omega^{3} v}{2\left(v_{A}^{4}+\omega^{2} v^{2}\right.}
$$

(ii) There is the magnetic diffusivity only. That is, there is no viscosity $(v=0)$. For this case, equation (4) gives

$k_{r}^{4}-P^{\prime} k_{r}^{2}-Q^{\prime 2}=0$ and $k_{r} k_{i}=Q^{\prime}$

where

$$
P^{\prime}=\frac{\omega^{2} v_{A}^{2}}{v_{A}^{4}+\omega^{2} \eta^{2}} \quad \text { and } \quad Q^{\prime}=\frac{\omega^{3} v}{2\left(v_{A}^{4}+\omega^{2} \eta^{2}\right)}
$$

DS finally calculated numerically the damping length scale $D$, (defined as $\left.D \equiv 2 \pi / k_{i}\right)$, group velocity $v_{g}$ (defined as $v_{g}=\partial \omega / \partial k$ ) and the energy flux density $W$ (defined as $W=\rho$ $v_{N T} v_{g}$ ) for three different frequencies. Here, $v_{\mathrm{NT}}$ is the velocity equivalent to the non-thermal component of the relevant spectral line at the full width at half maximum (FWHM).

\section{PHYSICAL PARAMETERS}

Investigation here requires information about various physical quantities. In the present investigation we also used the same expressions for (i) non-thermal component of velocity $v_{\mathrm{NT}}$, (ii) magnetic field $B$, (iii) electron density, $N_{\varepsilon}$, (iv) temperature $\mathrm{T}$, (v) magnetic diffusivity $\eta$, and (vi) viscosity coefficient $v$ as used by DS. However, their expressions for electron density and temperature need to be improved. After using their expressions, we have calculated values of $N_{\varepsilon}, T, v, \eta, B, v_{A}$ and $v_{\mathrm{NT}}$ in SI units as a function of $R$. The calculated values are given in Table $\mathbf{1}$.

\section{DISCUSSION}

In their investigation, DS accounted for three values of frequency $\omega\left(\equiv 2 \pi / \tau_{\mathrm{a}}\right)$ where they used $\tau_{\mathrm{a}}=0.01 \mathrm{~s}, 0.001 \mathrm{~s}$ and $0.0001 \mathrm{~s}$. Let us consider the two cases discussed by DS. 
Table 1. Physical Parameters

\begin{tabular}{|c|c|c|c|c|c|c|c|}
\hline$R^{a}$ & $N_{\varepsilon}^{b}$ & $T^{c}$ & $v^{d}$ & $\eta^{e}$ & $B^{f}$ & $v_{A}^{g}$ & $v_{N T}^{h}$ \\
\hline 1.05 & 6.79 & 0.45 & 0.20 & 3.79 & 9.51 & 3.25 & 3.35 \\
\hline 1.09 & 5.04 & 0.74 & 0.93 & 1.80 & 8.22 & 3.26 & 4.00 \\
\hline 1.11 & 4.36 & 0.86 & 1.56 & 1.44 & 7.64 & 3.26 & 4.23 \\
\hline 1.15 & 3.29 & 1.05 & 3.44 & 1.06 & 6.58 & 3.23 & 4.53 \\
\hline 1.17 & 2.87 & 1.12 & 4.65 & 0.96 & 6.10 & 3.21 & 4.62 \\
\hline 1.19 & 2.51 & 1.18 & 6.01 & 0.89 & 5.66 & 3.18 & 4.68 \\
\hline 1.21 & 2.20 & 1.22 & 7.46 & 0.85 & 5.26 & 3.16 & 4.72 \\
\hline 1.27 & 1.50 & 1.24 & 11.50 & 0.83 & 4.26 & 3.10 & 4.75 \\
\hline 1.29 & 1.32 & 1.22 & 12.40 & 0.85 & 4.00 & 3.10 & 4.76 \\
\hline 1.31 & 1.17 & 1.18 & 12.88 & 0.89 & 3.78 & 3.11 & 4.76 \\
\hline 1.33 & 1.04 & 1.12 & 12.86 & 0.96 & 3.60 & 3.14 & 4.78 \\
\hline 1.35 & 0.92 & 1.05 & 12.26 & 1.06 & 3.45 & 3.20 & 4.80 \\
\hline
\end{tabular}

${ }^{a}$ Distance from the center of sun in $\mathrm{R}_{\odot}$

${ }^{b}$ Electron density in $10^{13} \mathrm{~m}^{-3}$

${ }^{c}$ Temperature in $10^{6} \mathrm{~K}$

${ }^{d}$ Coefficient of viscosity in $10^{11} \mathrm{~m}^{2} \mathrm{~s}^{-1}$;

${ }^{e}$ Magnetic diffusivity in $\mathrm{m}^{2} \mathrm{~s}^{-1}$

${ }^{f}$ Magnetic field in $10^{-4} \mathrm{~T}$

${ }^{8}$ Alfvén velocity in $10^{6} \mathrm{~m} \mathrm{~s}^{-1}$

${ }^{h}$ Non-thermal velocity in $10^{4} \mathrm{~m} \mathrm{~s}^{-1}$

\subsection{Case of the Viscosity Only}

For $\eta=0$ (equation 5), we have

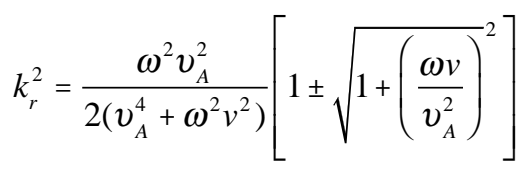

Using the data given in Table 1, we have $\omega v \gg v_{A}^{2}$ and we get

$$
k_{r}=\sqrt{\frac{\omega}{2 v}} \text { and therefore, } k_{i}=\sqrt{\frac{\omega}{2 v}}
$$

It shows that the damping length scale $D$ and the wavelength $\lambda$ of the wave are equal. For $\eta=0$ and $\omega v \gg>$ $v_{A}^{2}$, we have the group velocity

$$
v_{g} \equiv\left(\frac{\partial \omega}{\partial k}\right)_{r}=\sqrt{2 \omega v}
$$

This behaviour of the group velocity is quite different from that given by DS. For this group velocity, the energy flux density $W=\rho v_{N T} \sqrt{2 \omega v}$.

\subsection{Case of the Magnetic Diffusivity Only}

For $v=0$ (equation 6), we have

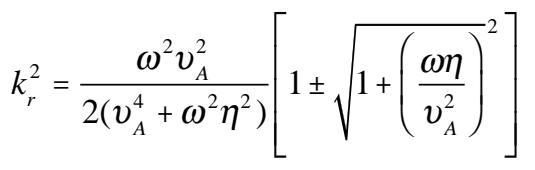

Using the data given in Table 1, the damping length scale $D$ and the wavelength $\lambda$ of the wave are

$$
D=\frac{2 \pi}{k_{i}}=\frac{4 \pi v_{A}^{3}}{\omega^{2} \eta} \text { and } \lambda=\frac{2 \pi}{k_{r}}=\frac{2 \pi v_{A}}{\omega}
$$

For $v=0$ and $\omega \eta \ll v_{A}^{2}$, we have the group velocity

$$
v_{g} \equiv\left(\frac{\partial \omega}{\partial k}\right)_{r}=v_{A}
$$

Hence, the energy flux density $W=\rho v_{N T} v_{A}$.

The analytical treatment of above two cases remains valid for $\tau_{a}=0.001$ and 0.0001 . For $\tau_{a}=0.01$, there may be some deviation from the above results obtained analytically. Values of the damping length scale, group velocity and the energy flux density for $v=0$ and $\eta=0$, separately, are given in Fig. (1). 

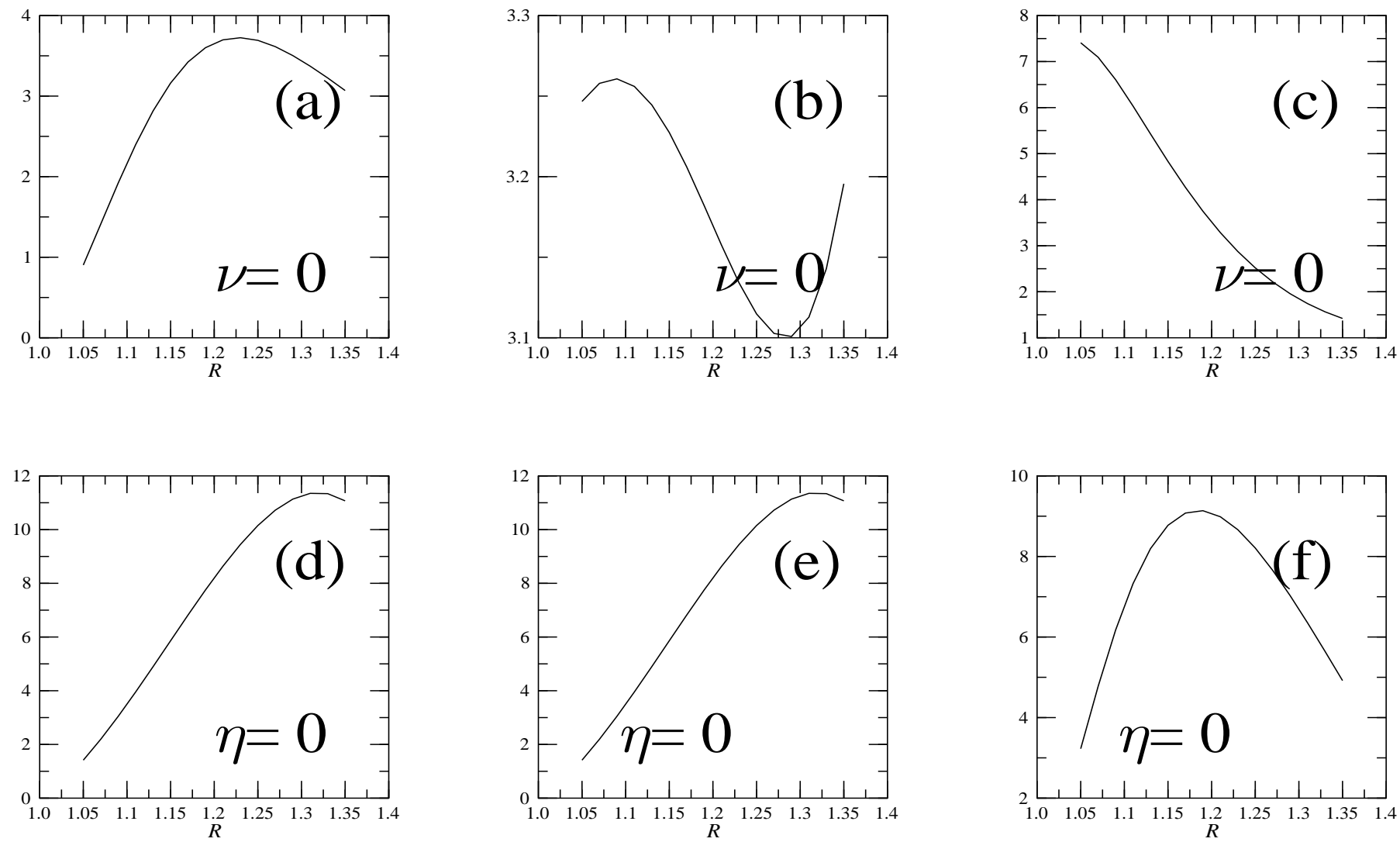

Fig. (1). Here, (a), (b) and (c) are for $v=0$ whereas (d), (e) and (f) are for $\eta=0$. (a) is $\_\omega^{2} D / 4 \pi$ in $10^{19} \mathrm{~m} \mathrm{~s}^{-2}$, (b) is $v_{\mathrm{g}}$ in $10^{6} \mathrm{~m} \mathrm{~s}^{-1}$, (c) is $W$ in $10^{-3} \mathrm{~kg} \mathrm{~m}^{-1} \mathrm{~s}^{-2}$, (d) is $D / \sqrt{8 \pi^{2} / \omega}$ in $10^{5} \mathrm{~m} \mathrm{~s}^{-1 / 2}$, (e) is $v_{g} / \sqrt{2 \omega}$ in $10^{5} \mathrm{~m} \mathrm{~s}^{-1 / 2}$ and (f) is $W / \sqrt{2 \omega}$ in $10^{-4} \mathrm{~kg} \mathrm{~m}^{-1} \mathrm{~s}^{-3 / 2}$.

\section{CONCLUSIONS}

Comparison of the results given in Fig. (1) with those reported by DS shows that their results are not reliable. Even there is no similarity between them. In particular, for the case (i), we found that the damping length scale is equal to the wavelength of the wave. For the case (ii), the group velocity as well as the energy flux density are found independent of the frequency. These findings contradict the results of DS. We tried to find out some cause for discrepancy. But it could be possible as DS did not provide any values for physical parameters, as we did by reporting in Table 1 here. Some reason for the difference in our results and those of DS can be assigned to some mistakes in their computer program or to the values of parameters used.

\section{ACKNOWLEDGMENTS}

We are thankful to the Department of Science \& Technology, New Delhi and the Indian Space Research
Organization (ISRO), Bangalore for financial support in the form of research projects. This work was done during visit to IUCAA, Pune as Visiting Associate of the Centre.

\section{REFERENCES}

[1] Dwivedi BN, Srivastava AK. On the propagation and dissipation of Alfvén waves in coronal holes. Solar Phys 2006; 237: 143-52.

[2] Parker EN. Dynamical theory of the solar wind. Space Sci Rev 1965; 4: 666-708.

[3] Hassler DM, Rottman GJ, Shoub EC, Holzer TE. Line broadening of MgX609 and $625 \AA$ coronal emission lines observed above the solar limb. Astrophys J 1990; 348: L77 -80.

[4] Harrison RA, Hood AW, Pike CD. Off-limb EUV line profiles and the search for wave activity in the low corona. Astron Astrophys 2002; 392: 319-27.

[5] Pekünlü ER, Bozkurt Z, Afsar M, Soydugan E, Soydugan F. Alfvén waves in the inner polar coronal hole. Mon Notices Roy Astron Soc 2002; 336: 1195-2000. 